Relations industrielles

Industrial Relations

\title{
Loi concernant la convention collective dans l'industrie de la construction (bill 90)
}

\section{Fernand Morin}

Volume 17, numéro 3, juillet 1962

URI : https://id.erudit.org/iderudit/1021577ar

DOI : https://doi.org/10.7202/1021577ar

Aller au sommaire du numéro

Éditeur(s)

Département des relations industrielles de l’Université Laval

ISSN

0034-379X (imprimé)

1703-8138 (numérique)

Découvrir la revue

Citer ce document

Morin, F. (1962). Loi concernant la convention collective dans l'industrie de la construction (bill 90). Relations industrielles / Industrial Relations, 17(3),

321-327. https://doi.org/10.7202/1021577ar
Résumé de l'article

La portée de la loi et le précédent qu'elle crée.
Tous droits réservés @ C Département des relations industrielles de l’Université Laval, 1962
Ce document est protégé par la loi sur le droit d'auteur. L'utilisation des services d'Érudit (y compris la reproduction) est assujettie à sa politique d'utilisation que vous pouvez consulter en ligne.

https://apropos.erudit.org/fr/usagers/politique-dutilisation/ 


\title{
COMMENTAIRES
}

\section{LOI CONCERNANT LA CONVENTION COLLECTIVE DANS L'INDUSTRIE DE LA CONSTRUCTION (bill 90)}

\author{
La portée de la loi et le précédent qu'elle crée
}

\section{FERNAND MORIN}

Le but de cette loi nous disent les «notes explicatives 》 du bill 90 , se divise en trois parties dont les deux dernières dépendent de la mise en vigueur de la première partie. Cette loi permet de:

1.- Extensionner, en vertu de la Loi de la convention collective, une clause d'une convention collective défendant le lockout, la grève et le piquetage;

2.- Soustraire les employeurs et les employés de l'observance des articles 4 - 17 de la Loi des relations ouvrières. Ainsi, l'employeur ne serait plus obligé de négocier une convention collective particulière durant l'existence d'un tel décret;

3.- Conférer au comité paritaire chargé de l'administration d'un tel décret, le pouvoir d'obliger les salariés, avec certaines exceptions, à obtenir un certificat de contrôle et à lui verser un certain honoraire.

\section{LE BUT DE LA LOI}

Avant d'analyser cette nouvelle législation, il est nécessaire de s'attarder quelque peu sur l'état actuel des relations ouvrières dans l'industrie de la construction. Rappelons d'abord que l'industrie de la construction du Canada et des Etats-Unis éprouve des difficultés énormes lors de l'application des lois ouvrières. Le caractère de cette industrie est essentiellement différent des autres. En effet, l'industrie de la construction est saisonnière et dans un mouvement constant. Chaque nouvelle saison signifie tantôt une augmentation, tantôt une diminution allant jusqu'à $30 \%$ de sa production. Sauf pour un nucléus d'employés attachés à chacun des entrepreneurs, il n'y a pas d'ouvriers engagés sur une base permanente dans l'industrie de la construction. Le lieu de travail n'est jamais le même; l'ouvrier restera deux, trois, ou quatre mois sur le même chantier pour se rendre ensuite sur les lieux d'une nouvelle construction. Dans bien des métiers spécialisés, l'ouvrier travaille durant la même année sur cinq ou six chantiers et change bien souvent autant de fois d'employeur. Dans une telle situation, comment les parties intéressées peuvent-elles établir de solides relations patronales-ouvrières? Il suffit de lire les conditions édictées pour l'obtention 
d'un certificat en reconnaissance syndicale pour comprendre que sauf dans des cas très exceptionnels, il est pratiquement impossible à un syndicat d'y satisfaire.

D'autre part, les statistiques démontrent que dans l'industrie de la construction, le Québec est la province qui compte le moins de lockout et de grève durant les vingt dernières années. Cette paix industrielle quasi-parfaite est certes un des heureux effets de la Loi de la convention collective, loi inexistante dans les autres provinces.

Parce que tous les ouvriers de la construction, membres ou non d'un syndicat, bénéficient en vertu des conventions collectives extensionnées, des mêmes conditions de travail, le recrutement des syndicats est devenu pénible et bien peu efficace. Pour cette rasion, le mouvement ouvrier a voulu ctte année faire un bris à la méthode actuelle des négociations collectives. Ainsi, ces syndicats, appuyés par certaines associations patronales, proposèrent au législateur un moyen pour remédier au problème du recrutement. Le bill 90 veut être la réponse de législateur.

Le but de nos remarques n'est pas de critiquer péjorativement cette loi; bien au contraire, même si nous contestons le moyen proposé, nous sommes heureux de constater que le gouvernement du Québec a bien voulu s'arrêter à ce problème de l'industrie de la construction et essayer d'y trouver une solution. Il nous faut espérer maintenant que d'ici la mise en vigueur du Code du Travail, l'expérience apportée par cette nouvelle loi (bill 90) saura aider toutes les parties intéressées à trouver la vraie solution. Pour nous, cette solution ne doit pas consister à éliminer une loi au bénéfice d'une autre, mais bien à trouver un moyen pour assurer la co-existence et l'efficacité des deux lois régissant actuellement les relations patronales-ouvrières: Loi des relations ouvrières Loi de la convention collective.

\section{ETUde sÉPARÉE des ARTICLes DE CETTE LOI}

Cette nouvelle loi a pour effet d'amender la Loi de la convention collective en y ajoutant trois articles. Voyons-les et discutons surtout des effets pratiques que peuvent produire ces trois articles.

\section{ARTICLE $10 a$}

«Dans l'industrie de la construction, le décret peut rendre obligatoire une disposition de la convention stipulant l'interdiction de grève, contre-grève, ralentissement de travail et piquetage. »

Disons d'abord que ce premier paragraphe de l'article 10a sert de démarreur pour déclencher l'application du deuxième paragraphe du même article et de l'article $20 \mathrm{~b}$. Ce premier paragraphe prévoit que les parties, si elles veulent profiter de cette nouvelle loi, doivent d'abord inclure dans leur convention collective à but d'extension, une clause interdisant la grève, la contre-grève, le ralentissement de travail et le piquetage. 
Pouvons-nous et surtout, devons-nous insérer une telle clause dans une convention collective? Rappelons que la très grande majorité des ouvriers en construction ne sont pas syndiqués et que les groupes de salariés pour lesquels un syndicat est certifié sont plutôt l'exception. Par conséquent, pourquoi convenir dans une convention collective à but d'extension une clause de non-grève puisque le nouvel article 24 de la Loi des relations ouvrières défend expressément au paragraphe premier: « ... toute grève et contre-grève... tant qu'une association de salariés n’a pas été reconnue comme représentant du groupe de salariés en cause... ».

Même pour les quelques groupes pour lesquels le syndicat est certifié, il semble inutile pour les parties d'inclure une telle clause dans la convention puisque ce même article 24 , au paragraphe 4 , stipule que: 《 ... toute grève ou contre-grève est interdite en toute circonstance pendant la durée d'une convention collective». En conséquence, dans ce domaine de grève et de contre-grève que le décret contienne ou non cette clause prohibitive, la grève ou contre-grève demeure et demeurera encore légale uniquement pour les conflits d'intérêt et seulement si le syndicat est certifié; ceci est vrai tant pour l'industrie de la construction que pour les autres industries. L'intérêt de faire inclure une telle clause dans un décret n'est donc pas pour protéger les parties d'une grève ou d'une contre-grève, mais bien pour pouvoir profiter du deuxième paragraphe de l'article 10a et de l'article $20 \mathrm{~b}$, qui en sont les conséquences édictées par le législateur et non pas l'effet causal.

Il appert à la lecture de ce premier paragraphe que tout le mécanisme qui peut déclencher une telle clause prohibitive dans le décret est basé sur un consentement préalable des parties intéressées. En effet, on exige la signature d'une convention collective comprenant telle prohibition. Demandons-nous alors si vraiment les parties sont libres de convenir d'une telle clause? Supposons qu'une des deux parties s'y refuse: rendu à l'arbitrage, il est fort probable que la décision arbitrale recommanderait l'insertion d'une telle clause. En effet, comment ce tribunal peut-il refuser de recommander ce que le législateur lui-même impose $\ll \ldots$ en toute circonstance pendant la durée d'une convention collective ». (Bill 78, article 24, paragraphe 4) Avec des pressions semblables, peut-on réellement prétendre que les parties sont réellement libres d'y consentir ou non?

Cet article 10a permet candidement à l'employeur et au syndicat de ses employés, association généralement non reconnue par la Commission des Relations Ouvrières, de convenir sur l'interdiction $d u$ ralentissement de travail et de piquetage. Soulignons que déjà l'article 25 de la Loi des relations ouvrières prévoit cette interdiction de ralentissement de travail ou grève perlée. Le législateur provincial peut-il d'une façon si simpliste, s'en remettre aux parties intéressées pour défendre un acte dont la légalité est reconnue et protégée par le code criminel: $366(2)$ «Ne surveille ni ne cerne, au sens du présent article, celui qui est présent à ou près une maison d'habitation ou un lieu, ou s'en appro- 
che à seule fin d'obtenir ou de communiquer des renseignements ». Nous ne traiterons pas ici les problèmes de droit constitutionnel soulevés par cet article à savoir par exemple les cas et la façon dont une législation provinciale peut prohiber un acte expressément permis et protégé par une loi fédérale. Soulignons également que ce n'est pas le législateur québécois qui prohibe lui-même le piquetage, il se contente de permettre aux deux parties intéressées de convenir sur cette prohibition et s'en remet au Lieutenant-Gouverneur en conseil pour exetnsionner une telle clause.

Qu'est-ce que le piquetage? Souvent on le définit comme suit: Une pratique adoptée par les salariés engagés dans un différend ouvrier, et qui consiste à placer des hommes sur le chantier de l'employeur récalcitrant, dans le but d'obtenir de lui ou de lui arracher des informations sur l'objet en dispute. Il peut également avoir pour but de renseigner sur un point donné (un grief) le public ou l'ensemble des salariés de l'employeur. Disons à titre d'illustration, que lors d'un grief sur l'interprétation de la convention collective, le syndicat désire informer tous les travailleurs du chantier de construstion de l'existence de ce différend. L'interdiction d'un piquetage ne permettrait plus alors au syndicat de placer à l'entrée du chantier une personne portant une pancarte pour indiquer ces faits. Au dire de l'article $45 \mathrm{a}$, cette personne «... commet un acte illégal et est passible d'une amende n'excédant pas cent dollars et les frais... » Il est reconnu par la jurisprudence qu'un piquetage pacifique peut exister seul sans être nécessairement l'instrument d'une grève. Comment peut-on interdire, par un simple paragraphe laissant pleine discrétion aux parties, un acte dont la légalité est expressément reconnue par une loi et d'autre part, ne nuit et n'est préjudiciable à personne?

\section{ARTICLE $10 a$ - deuxième paragraphe}

\& Le présent décret, en ce cas, a pour effet de suspendre pour toute sa durée, pour les employeurs et salariés qu'il régit et leurs associations, l'application des articles 4 à 17 de la Loi des relations ouvrières (chap. 162A).

Ainsi, pour la durée du décret, aucun syndicat ne peut faire une requête en reconnaissance syndicale et d'autre part, l'association déjà certifiée perd momentanément ses privilèges. Ce deuxième paragraphe de l'article 10a essaie de répondre à une requête des employeurs qui se sont toujours objectés à la double négociation collective. Je crois que cette suspension des articles 4 à 17 préserve théoriquement seulement cette double négociation, c'est-à-dire la négociation d'une convention collective particulière accordant des conditions de travail supérieures à celles prévues au décret. En effet, l'article 18 de la Loi des relations ouvrières demeure et permet toujours la signature de conventions collectives particulières avec des associations non-reconnues. Or, il s'avère que dans l'industrie de la construction, c'est justement ce genre de conventions qui sont signées par les parties. Certes, l'entrepreneur n'est plus légalement forcé de «... négocier, de bonne foi avec eux, une con- 
vention collective de travail... $\gg$ (article 4 de la Loi des relations ouvrières) mais en pratique, c'est une toute autre affaire. Un entrepreneur qui exécute de temps à autres des travaux en dehors de la province, se voit bien souvent obligé par la force des circonstances, à signer une convention collective pour tous ses employés présents et futurs, y compris ceux du Québec, s'il désire obtenir le personnel nécessaire pour l'exécution de ses travaux à l'extérieur de la province. Sans développer plus à fond ce sujet, rappelons que la majorité des gros entrepreneurs généraux ou spécialisés ont déjà de telles conventions et devront sans aucun doute continuer à en signer. Le bill 90 n'arrêtera certes pas ces pressions économiques.

\section{Certifications}

L'application des articles 4 à 17 est suspendue pour la durée seulement du décret. Conséquemment, un syndicat peut, du moins théoriquement, demander sa certification au terme d'un tel décret. Pour ce faire, il faudra que ce décret cesse complètement, qu'il soit dénoncé catégoriquement par les parties et que la période intermédiaire entre la fin d'un décret et le début de l'autre ne soit pas couverte par un décret de prolongation. D'autre part, les effectifs des syndicats seront, en vertu de l'application de l'article 20b, grandement augmentés, ce qui peut avoir pour effet d'inciter les syndicats à provoquer une période creuse c'est-à-dire où il n'y aura pas de décret en vigueur, pour pouvoir demander leur reconnaissance syndicale auprès de la Commission des Relations Ouvrières.
ARTICLE $20 \mathrm{~b}$
« Dans une région soumise à un décret rendant obligatoire une con- vention stipulant l'interdiction visée à l'article $10 \mathrm{a}$ et où est en activité une commission d'apprentissage de métiers de la construction, le comité peut, par règlement approuvé par le lieutenant-gouverneur en conseil, obliger, avec les exceptions qu'il juge à propos, les salariés à obtenir périodiquement un certificat de contrôle pour fins de sur- veillance de l'apprentissage ou de la compétence professionnelle, et à verser à titre d'honoraire mensuel, une somme n'excédant pas le salaire horaire fixé par le décret pour leur catégorie. Déduction faite des frais d'administration, les sommes ainsi perçues sont versées à telle commission d'apprentissage pour être employées aux fins indi- quées dans le présent article.

Ainsi, selon le texte de cet article, le comité paritaire peut exercer un certain contrôle de l'apprentissage et de la compétence professionnelle des travailleurs de la construction. Analysons d'abord cet article en prenant seulement en considération le texte tel que rédigé et selon sa signification normale. Après, nous pourrons considérer ce que cet article signifie à toute fin pratique ou encore, la façon dont ses promoteurs veulent le comprendre et l'utiliser.

Cet article 20b a pour effet d'accroître considérablement la juridiction du comité paritaire. Jusqu'ici le comité conjoint avait pour prin- 
cipale et quasi unique mission «... d'assurer l'observance du décret, de ses modifications et de ses renouvellements ». (article 16, chap. 163) L'article 20 de la même loi énumère une série de droits et de pouvoirs conférés à ce comité paritaire pour qu'il puisse effectivement contrôler l'observance du décret. Ainsi, en vertu de cet article 20, le comité peut vérifier dans les livres mêmes des employeurs, les noms et qualifications de tous les employés et obliger les employeurs à lui transmettre copie de la liste de leur personnel. Le comité était donc jusqu'ici limité à surveiller l'observance de ce qui était stipulé dans la convention collective extensionnée. Maintenant, en vertu du nouvel article $20 \mathrm{~b}$, il semble qu'on élargit les cadres de la juridiction du comité. En effet, le comité pourra dorénavant non plus se limiter au décret mais aussi, exercer une surveillance sur l'apprentissage et sur la compétence professionnelle de tous les travailleurs de la construction. Il est bon de se rappeler ici que déjà le gouvernement exerce pour plusieurs métiers, un contrôle efficace de l'apprentissage et de la compétence professionnelle des travailleurs: Loi des Electriciens et des installations électriques, chap. 172, S.R.Q. 1941 - Loi des mécaniciens en tuyauterie, chap. 173, S.R.Q. 1941.

Pour plusieurs autres métiers, la Commission d'Apprentissage exerce actuellement ce contrôle, au moins dans la région de Montréal. En plus de verser des honoraires au gouvernement pour le coût de cette surveillance (du moins pour les plombiers et les électriciens) et de verser $1 / 2$ de $1 \%$ de leur salaire au comité conjoint dont une partie de cet argent sert déjà à exercer un contrôle, les ouvriers de la construction devront maintenant payer mensuellement d'autres honoraires pour ce même contrôle.

\section{Formule Rand}

Maintenant, regardons ce même article dans l'optique de ses promoteurs. L'article $20 \mathrm{~b}$ stipule que le «comité peut, par règlement approuvé par le lieutenant gouverneur en conseil, obliger, avec les exceptions qu'il juge à propos, ... » Quels groupes de salariés seront-ils compris dans ces exceptions? Il nous semble normal d'y inclure les travailleurs déjà sous une surveillance directe d'un Bureau d'Examinateurs du gouvernement: c'est le cas des électriciens et des plombiers. D'après les protagonistes de ce bill, seraient exclus de cette surveillance du comité paritaire, les travailleurs membres en règle d'un syndicat. Il faut croire alors que le syndicat ferait lui-même cette surveillance. Les syndicats ouvriers en ont-ils la capacité et l'autorité? Si c'est le cas, c'est-à-dire si les travailleurs exclus du contrôle du comité paritaire, sont ceux membres d'un syndciat, nous avons alors de par cet article $20 \mathrm{~b}$, l'application d'une formule Rand mitigée. Le non-syndiqué, le franc-tireur ou black-legs, paierait à un tiers, sous forme d'honoraires, une somme d'argent d'un montant sensiblement égal à celui que paie le syndiqué sous forme cette fois de cotisation. La question se pose alors à savoir s'il est souhaitable que le législateur s'ingère dans un tel 
domaine et influence d'une façon indirecte les travailleurs à faire partie d'une association. Est-ce vraiment là ce qưon peut appeler le respect de la liberté d'association?

Jusqu'ici l'Etat a toujours joué le rôle d'arbitre entre le patron et l'ouvrier, il servait bien souvent de tampon, de conciliateur et règlementait la procédure des pourparlers. Aujourd'hui le législateur semble, en vertu de ce bill 90, vouloir prendre d'une façon indirecte la position d'agent d'affaires du syndicat ouvrier!

\section{Conclusion}

Ces quelques remarques n'ont certes pas vidé la question posée par cette nouvelle législation. Nous espérons que des personnes plus qualifiées prendront la relève et sauront défendre comme il se doit les intéressés et le public en général.

Si mes quelques remarques savent inciter les autorités en la matière à étudier le problème et à proposer des solutions plus équitables, j’aurai atteint mon but.

\section{JURISPRUDENCE DU TRAVAIL}

\section{SERVICES PUBLICS - POUVOIR DU TRIBUNAL D'ARBITRAGE D'AMENDER SA PROPRE SENTENCE}

A council of arbitration, appointed under the Act respecting Municipal and School Corporations and their Employees, which remained in office after rendering its award with power to hear any dispute arising as to its interpretation but whose authority has been limited by a clause in the agreement annexed to the award and reading as follows:

Le Tribunal d'arbitrage n'aura pas juridiction pour rendre une décision incompatible avec les dispositions de cette convention, ni pour changer, modifier ou amender quelque partie que ce soit de cette convention.

has the right to interpret its award and to correct a simple clerical error, but not to amend it.

In the present instance, under the terms of the award, the employees of the same category as plaintiff were entitled to be paid at the rate of $\$ 1.29$ per hour for time worked up to 44 hours a week, pius 50\% for time worked in excess of 44 hours a week, the whole retroactive to a specified date. It cannot be said that it is only through a clerical error that the award was made retroactive not only 